\title{
Influence of IL-18 and IL-10 Polymorphisms on Tacrolimus Elimination in Chinese Lung Transplant Patients
}

\author{
Xiaoqing Zhang, ${ }^{1}$ Jiandong Xu, ${ }^{2}$ Junwei Fan, ${ }^{3}$ Tao Zhang, ${ }^{3}$ Yuping Li, ${ }^{1}$ Boxiong Xie, \\ Wei Zhang, ${ }^{1}$ Shengtao Lin, ${ }^{3}$ Ling Ye, ${ }^{3}$ Yuan Liu, ${ }^{3}$ and Gening Jiang ${ }^{4}$ \\ ${ }^{1}$ Department of Pharmacy, Shanghai Pulmonary Hospital, Tongji University School of Medicine, Shanghai, China \\ ${ }^{2}$ Department of Pharmacy, Jiangwan Hospital of Shanghai Hongkou District, Shanghai, China \\ ${ }^{3}$ Department of General Surgery, Shanghai First People's Hospital, Shanghai Jiaotong University, Shanghai, China \\ ${ }^{4}$ Department of Thoracic Surgery, Shanghai Pulmonary Hospital, Tongji University School of Medicine, Shanghai, China
}

Correspondence should be addressed to Junwei Fan; fjwnet@163.com and Gening Jiang; jgn1121@163.com

Received 30 July 2016; Accepted 1 November 2016; Published 26 January 2017

Academic Editor: Gad Rennert

Copyright (c) 2017 Xiaoqing Zhang et al. This is an open access article distributed under the Creative Commons Attribution License, which permits unrestricted use, distribution, and reproduction in any medium, provided the original work is properly cited.

\begin{abstract}
Aims. The influence of interleukin-10 (IL-10) and interleukin-18 (IL-18) polymorphisms on tacrolimus pharmacokinetics had been described in liver and kidney transplantation. The expression of cytokines varied in different kinds of transplantation. The influence of IL-10 and IL-18 genetic polymorphisms on the pharmacokinetic parameters of tacrolimus remains unclear in lung transplantation. Methods. 51 lung transplant patients at Shanghai Pulmonary Hospital were included. IL-18 polymorphisms (rs5744247 and rs1946518), IL-10 polymorphisms (rs1800896, rs1800872, and rs3021097), and CYP3A5 rs776746 were genotyped. Dose-adjusted trough blood concentrations ( $C / D$ ratio, $\mathrm{mg} / \mathrm{kg}$ body weight) in lung transplant patients during the first 4 postoperative weeks were calculated. Results. IL-18 rs5744247 allele C and rs1946518 allele A were associated with fast tacrolimus metabolism. Combined analysis showed that the numbers of low IL-18 mRNA expression alleles had positive correlation with tacrolimus $C / D$ ratios in lung transplant recipients. The influence of IL-18 polymorphisms on tacrolimus $C / D$ ratios was observed in CYP3A5 expresser recipients, but not in CYP3A5 nonexpresser recipients. No clinical significance of tacrolimus $C / D$ ratios difference of IL-10 polymorphisms was found in our data. Conclusions. IL-18 polymorphisms may influence tacrolimus elimination in lung transplantation patients.
\end{abstract}

\section{Introduction}

Lung transplantation is the ultimate treatment option for those with end-stage lung diseases. Unfortunately, the median survival time after lung transplantation is just over 6 years [1-3]. Long-term patient and graft survival are limited by both acute and chronic lung allograft rejection. Current immunosuppression regimen lifelong used in the majority of lung transplant recipients comprised a calcineurin inhibitor (e.g., tacrolimus), an antimetabolite (e.g., mycophenolate mofetil), and corticosteroids [4]. Tacrolimus binding to a cytoplasmic protein, FK506-binding protein 12 (FKBP12), formed a complex. This complex inhibited transcription of cytokines, including interleukin-2, and ultimately blocked T-lymphocyte activation and proliferation.
Tacrolimus-based immunosuppressive therapy was characteristic of narrow therapeutic index and broad interindividual variability. Underimmunosuppression resulted in allograft loss while overimmunosuppressive therapy caused infection, lymphoproliferative disease, and multiple drug toxicities (e.g., renal dysfunction, diabetes mellitus, dyslipidemia, obesity, and arterial hypertension) [5, 6]. Therefore, tailoring immunosuppression to an individual patient might improve the outcome of lung transplantation.

Pharmacogenetics, which is the study of genetic differences affecting individual responses to drugs, might help to optimize the initiation and maintenance dosage of tacrolimus to reach its target concentrations rapidly and to reduce its adverse reactions $[7,8]$. At present, several single nucleotide polymorphisms (SNPs) have been studied in relation to 
the dosing of tacrolimus [9]. The predominant enzyme responsible for tacrolimus metabolism is CYP3A5 and the CYP3A5 A6986G polymorphism had a strong influence on tacrolimus elimination $\left({ }^{*} 3\right)$. The variant sequences $A \rightarrow G$ cause alternative splicing and the formation of a truncated CYP3A5 protein that is not functional. Consequently, individuals with homozygous allele CYP3A5*3 (6986 GG) are often referred to as "CYP3A5 nonexpressers." Patients with at least one CYP3A5 ${ }^{*} 1$ wild-type allele (6986 GA/AA) are able to produce functional CYP3A5 enzymes and are known as "CYP3A5 expressers" [10]. The impact of CYP3A5 A6986G polymorphisms on tacrolimus dose requirements has been confirmed in liver, kidney, and lung transplantation studies [11-14]. Although the association between SNPs in CYP3A4, $\mathrm{ABCB} 1$, and other genes and tacrolimus elimination had been observed in numerous studies, research has been able to reliably show that only the CYP3A5 genotype can modify the pharmacokinetics of tacrolimus at present [15].

Cytokines are thought to be potent depressors of cytochrome P450 (CYP) enzymes and cause the depression of CYP-associated drug metabolism. The CYP gene expression and enzyme activities are downregulated during inflammation, infection, and transplantation rejection [16, 17]. The influence of interleukin-10 (IL-10) and interleukin18 (IL-18) polymorphisms on tacrolimus pharmacokinetics had been described in liver and kidney transplantation. $\mathrm{Li}$ et al. $[18,19]$ found that the IL-10 G-1082A polymorphism in recipients was significantly associated with tacrolimus concentration/dose $(C / D)$ ratios (IL-10-1082GG $<\mathrm{GA}<\mathrm{AA}$ ) within the first 3 weeks after liver transplantation; however, no significant effect of IL-10 G-1082A polymorphism was observed on population pharmacokinetic parameter of tacrolimus within 175 days after liver transplantation. Our previous study showed that the tacrolimus $C / D$ ratios of the recipients with donors who were CYP3A5 nonexpressers and had low IL-10 production genotype (-819TT, -592 AA) were higher than those with donors who were CYP3A5 nonexpressers and had high IL-10 production genotype (819CC or CT, $-592 \mathrm{CC}$ or AC) in the first 2 weeks after liver transplantation [20]. Another study showed that the proportion of patients in the IL-10-819 TT group who achieved the target $\mathrm{C} 0$ ranges was greater compared to the IL-10-819 CT/CC groups at week 3 after kidney transplantation [21].

The recipients with higher IL-18 serum levels had lower tacrolimus $C / D$ ratios in liver transplantation [22]. Donor IL-18 rs5744247 polymorphism was an independent predictor of tacrolimus elimination in the first week after liver transplantation resulting from two independent cohorts [23]. IL-18 rs1946518 gene polymorphism was associated with log-transformed tacrolimus $C / D$ ratios in Chinese kidney transplant recipients [24].

The target tacrolimus concentration, incidence rate of infection, and rejection in lung transplant recipients were higher than those in liver and kidney transplant recipients [46]. The expression of cytokines and tacrolimus metabolism varied in different kinds of transplantation. To date, the influence of IL-10 and IL-18 genetic polymorphisms on the pharmacokinetic parameters of tacrolimus remains unclear in lung transplantation.
In this study, we examined the IL-10 and IL-18 genotypes of lung transplant recipients to clarify the influence of these genetic variants on tacrolimus elimination after transplantation. We used the $C / D$ ratio as an index of tacrolimus pharmacokinetics.

\section{Patients and Methods}

A total of 51 (7 female and 44 male) adult lung transplant recipients who received tacrolimus-based immunosuppressive regimens at Shanghai Pulmonary Hospital between July 2005 and July 2015 were included in this study. Transplant subtypes included 29 single right lung transplants, 16 single left lung transplants, and 6 bilateral lung transplants. All patients were Han Chinese. The mean age $( \pm S D)$ was $54 \pm 10$ years (range: 28 to 75 years) and mean body weight was $58.5 \pm 13.3 \mathrm{~kg}$ (range: 36 to $86 \mathrm{~kg}$ ). Tacrolimus trough levels $C O$ $(\mathrm{ng} / \mathrm{mL})$ were determined by Pro-TracIITM FK506 ELISA kit (DiaSorin, USA) with microparticle enzyme immunoassay (ELx 800NB analyzer, BioTek, USA). Dose-adjusted trough blood concentrations ( $C / D$ ratio, $\mathrm{mg} / \mathrm{kg}$ body weight) in 4 weeks after transplantation were calculated. 68 normal (nondiseased) liver tissues were previously collected from Chinese donors in Shanghai First People's Hospital. The study was approved by the Ethics Committee of Shanghai Pulmonary Hospital and Shanghai First People's Hospital. Written informed consent was obtained from all patients in accordance with the Declaration of Helsinki and its amendments.

\subsection{Genomic DNA Isolation and Genotyping. CYP3A5} rs776746, 2 SNPs of IL-18 (rs5744247 and rs1946518), and 3 SNPs of IL-10 (rs1800896, rs1800872, and rs3021097) were genotyped. Peripheral blood samples were collected in ethylenediaminetetraacetic acid (EDTA) tubes and preserved at $-20^{\circ} \mathrm{C}$ before use. DNA was extracted from whole blood using a standard phenol-chloroform method. A multiplexed SNP MassEXTEND assay was designed by the Sequenom MassARRAY Assay Design 3.0 software (San Diego, CA, USA). The 6 SNPs were genotyped using the Sequenom MassARRAY RS1000 according to the standard protocol recommended by the manufacturer. Data were managed using the Sequenom Typer 4.0 software. Hardy-Weinberg equilibrium, allele frequency, and linkage disequilibrium were analyzed using SHEsis software (http://analysis.biox.cn/myAnalysis.php) [25].

2.2. Real-Time Reverse Transcriptase-PCR. Total RNA was extracted using RNeasy kit (Qiagen, Hilden, Germany) following the manufacturer's protocol. First-strand cDNA was synthesized using High Capacity cDNA Reverse Transcription Kit according to the manufacturer's instruction (Applied Biosystems, Carlsbad, CA, USA). Quantitative PCR was performed with SYBR Green PCR Master Mix (Applied Biosystems) and Mastercycler ep realplex (Eppendorf, Hamburg, Germany). IL-18 was amplified using the sense primer $5^{\prime}$-CCAAGGAAATCGGCCTCTAT- $3^{\prime}$ and antisense primer $5^{\prime}$-TTGTTCTCACAGGAGAGAGTTGA$3^{\prime}$. $\beta$-Actin was amplified as endogenous control using 
TABLE 1: Allele frequency of CYP3A5, IL-18, and IL-10 polymorphisms in lung transplant recipients $(n=51)$.

\begin{tabular}{|c|c|c|c|c|c|c|}
\hline \multirow{3}{*}{$\begin{array}{l}\text { Gene } \\
\text { CYP3A5 }\end{array}$} & \multirow{3}{*}{$\begin{array}{c}\text { SNP } \\
\text { rs776746 }\end{array}$} & \multicolumn{3}{|c|}{ Genotype } & \multicolumn{2}{|c|}{ Allele } \\
\hline & & GG & $A G$ & AA & G & $\mathrm{A}$ \\
\hline & & $0.471(24)$ & $0.451(23)$ & $0.078(4)$ & $0.696(71)$ & $0.304(31)$ \\
\hline \multirow{2}{*}{ IL-18 } & \multirow{2}{*}{ rs5744247 } & GG & GC & $\mathrm{CC}$ & G & $\mathrm{C}$ \\
\hline & & $0.118(6)$ & $0.510(26)$ & 0.373 (19) & $0.373(38)$ & $0.627(64)$ \\
\hline \multirow{2}{*}{ IL-18 } & \multirow{2}{*}{ rs1946518 } & $\mathrm{CC}$ & $\mathrm{CA}$ & $\mathrm{AA}$ & $\mathrm{C}$ & $\mathrm{A}$ \\
\hline & & $0.255(13)$ & $0.451(23)$ & $0.294(15)$ & $0.480(49)$ & $0.520(53)$ \\
\hline \multirow{2}{*}{ IL-10 } & \multirow{2}{*}{ rs1800896 } & AA & AG & GG & A & G \\
\hline & & $0.863(44)$ & $0.137(7)$ & $0(0)$ & $0.931(95)$ & $0.069(7)$ \\
\hline \multirow{2}{*}{ IL-10 } & \multirow{2}{*}{ rs1800872 } & AA & $\mathrm{AC}$ & $\mathrm{CC}$ & A & $\mathrm{C}$ \\
\hline & & $0.412(21)$ & $0.510(26)$ & $0.078(4)$ & $0.667(68)$ & $0.333(34)$ \\
\hline \multirow{2}{*}{ IL-10 } & \multirow{2}{*}{ rs3021097 } & $\mathrm{TT}$ & $\mathrm{TC}$ & $\mathrm{CC}$ & $\mathrm{T}$ & $\mathrm{C}$ \\
\hline & & $0.431(22)$ & $0.490(25)$ & $0.078(4)$ & $0.676(69)$ & $0.324(33)$ \\
\hline
\end{tabular}

TABLE 2: Comparison of tacrolimus concentration/dose ratios in different groups of CYP3A5, IL-18, and IL-10 polymorphisms at different times after drug initiation.

\begin{tabular}{|c|c|c|c|c|c|c|c|c|c|c|}
\hline \multirow{2}{*}{ Gene } & \multirow{2}{*}{ Locus } & \multirow{2}{*}{ Genotype } & \multicolumn{2}{|c|}{ Week 1} & \multicolumn{2}{|l|}{ Week 2} & \multicolumn{2}{|c|}{ Week 3} & \multicolumn{2}{|c|}{ Week 4} \\
\hline & & & $C / D$ ratio & $P$ & $C / D$ ratio & $P$ & $C / D$ ratio & $P$ & $C / D$ ratio & $P$ \\
\hline \multirow{2}{*}{ CYP3A5 } & \multirow{2}{*}{ rs776746 } & GG & $243.9 \pm 244.2$ & \multirow{2}{*}{0.005} & $218.2 \pm 211.9$ & \multirow{2}{*}{0.008} & $194.0 \pm 212.2$ & \multirow{2}{*}{0.003} & $156.8 \pm 188.6$ & \multirow{2}{*}{$<0.001$} \\
\hline & & $\mathrm{AG}+\mathrm{AA}$ & $96.8 \pm 145.6$ & & $97.0 \pm 143.0$ & & $79.5 \pm 130.6$ & & $80.6 \pm 59.2$ & \\
\hline \multirow{2}{*}{ IL-18 } & \multirow{2}{*}{ rs5744247 } & $\mathrm{GG}+\mathrm{CG}$ & $202.3 \pm 241.1$ & \multirow{2}{*}{0.002} & $204.6 \pm 181.0$ & \multirow{2}{*}{0.004} & $192.2 \pm 186.7$ & \multirow{2}{*}{0.005} & $115.7 \pm 132.4$ & \multirow{2}{*}{0.026} \\
\hline & & $\mathrm{CC}$ & $92.4 \pm 144.2$ & & $79.2 \pm 135.5$ & & $74.8 \pm 133.8$ & & $73.9 \pm 110.3$ & \\
\hline \multirow{3}{*}{ IL-18 } & \multirow{3}{*}{ rs1946518 } & CC & $269.5 \pm 239.0$ & \multirow{3}{*}{0.007} & $224.0 \pm 192.4$ & \multirow{3}{*}{0.018} & $197.7 \pm 226.3$ & & $151.0 \pm 193.5$ & \multirow{3}{*}{0.079} \\
\hline & & $\mathrm{CA}$ & $191.6 \pm 192.2$ & & $159.3 \pm 194.6$ & & $158.7 \pm 158.6$ & 0.019 & $108.1 \pm 96.8$ & \\
\hline & & $\mathrm{AA}$ & $76.6 \pm 108.8$ & & $79.2 \pm 86.3$ & & $65.9 \pm 84.4$ & & $73.9 \pm 110.3$ & \\
\hline \multirow{2}{*}{ IL-10 } & \multirow{2}{*}{ rs1800896 } & AA & $193.9 \pm 224.8$ & \multirow{2}{*}{0.027} & $176.8 \pm 205.1$ & \multirow{2}{*}{0.034} & $170.8 \pm 186.7$ & \multirow{2}{*}{0.24} & $104.8 \pm 125.9$ & \multirow{2}{*}{0.24} \\
\hline & & $\mathrm{AG}$ & $77.2 \pm 66.4$ & & $74.1 \pm 32.2$ & & $77.8 \pm 41.6$ & & $85.6 \pm 44.9$ & \\
\hline \multirow{2}{*}{ IL-10 } & \multirow{2}{*}{ rs1800872 } & AA & $191.6 \pm 232.3$ & \multirow{2}{*}{0.985} & $167.5 \pm 222.7$ & \multirow{2}{*}{0.863} & $158.7 \pm 228.8$ & \multirow{2}{*}{0.909} & $98.8 \pm 156.3$ & \multirow{2}{*}{0.605} \\
\hline & & $\mathrm{CA}+\mathrm{CC}$ & $165.8 \pm 209.1$ & & $137.8 \pm 171.2$ & & $133.8 \pm 148.0$ & & $103.0 \pm 86.3$ & \\
\hline IL-10 & rs3021097 & $\mathrm{CC}+\mathrm{TC}$ & $159.2 \pm 211.7$ & 1000 & $138.7 \pm 179.2$ & 0.909 & $133.9 \pm 154.5$ & 0879 & $97.8 \pm 86.8$ & 0.634 \\
\hline & & $\mathrm{TT}$ & $184.3 \pm 211.5$ & & $145.5 \pm 217.2$ & & $134.6 \pm 224.2$ & & $100.0 \pm 142.5$ & \\
\hline
\end{tabular}

the sense primer $5^{\prime}$-GGGTCAGAAGGATTCCTATG- $3^{\prime}$ and the antisense primer $5^{\prime}$-GGTCTCAAACATGATCTGGG-3' [26]. These reactions were repeated three times.

2.3. Statistical Analysis. Experimental data were analyzed using SPSS version 17.0 (SPSS, USA) and GraphPad Prism version 5.00 (http://www.graphpad.com/) software. The tacrolimus $C / D$ ratios are expressed as median \pm interquartile range. The difference in $C / D$ ratios was compared using Mann-Whitney $U$ test or Kruskal-Wallis test among groups. $t$-tests were performed to compare IL-18 mRNA in different genotypes. The statistical graphs were made by GraphPad Prism version 5.00 software (http://www.graphpad.com/). Two-tailed $P$ values of less than 0.05 were considered statistically significant.

\section{Results}

3.1. Effect of Single SNP on Tacrolimus C/D Ratios. Allele and genotype frequency of the CYP3A5 rs776746, IL-18 rs5744247, IL-18 rs1946518, IL-10 rs1800896, IL-10 rs1800872, and IL-10 rs3021097 are shown in Table 1. All six SNPs were in accordance with Hardy-Weinberg equilibrium $(P>0.05)$. The effects of single donor SNP on tacrolimus $C / D$ ratios are shown in Table 2. Tacrolimus $C / D$ ratios of CYP3A5 rs776746 GG genotype carriers were $243.9 \pm 244.2,218.2 \pm 211.9$, $194.0 \pm 212.2$, and $156.8 \pm 188.6$ at week 1 , week 2 , week 3 , and week 4, respectively. For AG and AA genotype carriers, the corresponding tacrolimus $C / D$ ratios were $96.8 \pm 145.6$, $97.0 \pm 143.0,79.5 \pm 130.6$, and $80.6 \pm 59.2$. The differences were significant at week 1 , week 2 , week 3 , and week $4(P=0.005$, $0.008,0.003$, and $<0.001$, resp.). Tacrolimus $C / D$ ratios of IL18 rs5744247 GG+CG genotype carriers were $202.3 \pm 241.1$, $204.6 \pm 181.0,192.2 \pm 186.7$, and $115.7 \pm 132.4$ at week 1 , week 2 , week 3 , and week 4 , respectively. For CC genotype carriers, the corresponding tacrolimus $C / D$ ratios were $92.4 \pm 144.2$, $79.2 \pm 135.5,74.8 \pm 133.8$, and $73.9 \pm 110.3$. The differences were significant at week 1 , week 2 , week 3 , and week $4(P=$ $0.002,0.004,0.005$, and 0.026 , resp.). Tacrolimus $C / D$ ratios of IL-18 rs1946518 CC genotype carriers were $269.5 \pm 239.0$, 


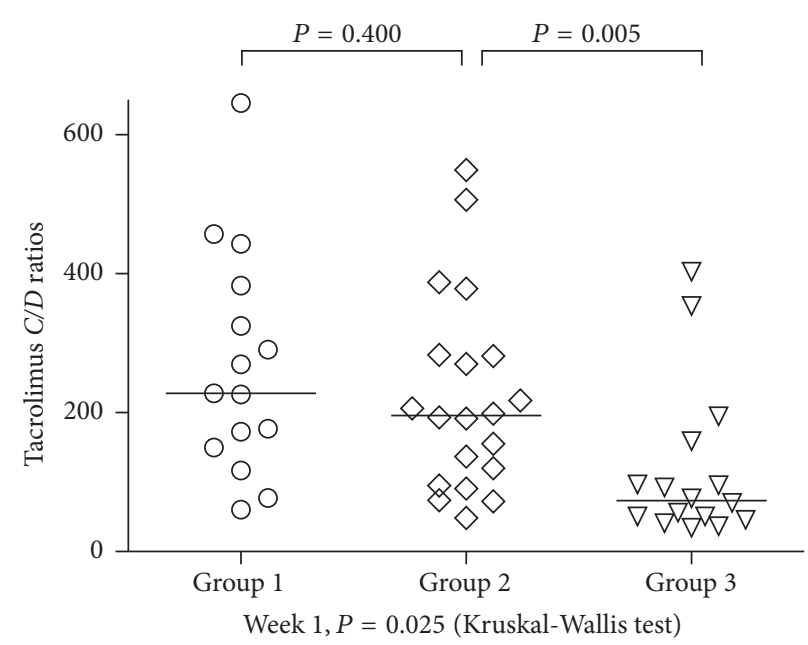

(a)

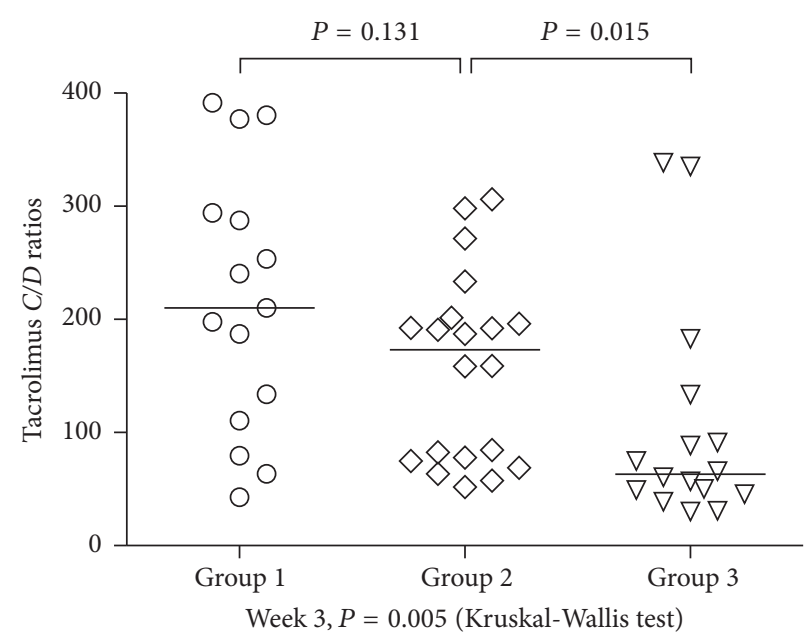

(c)

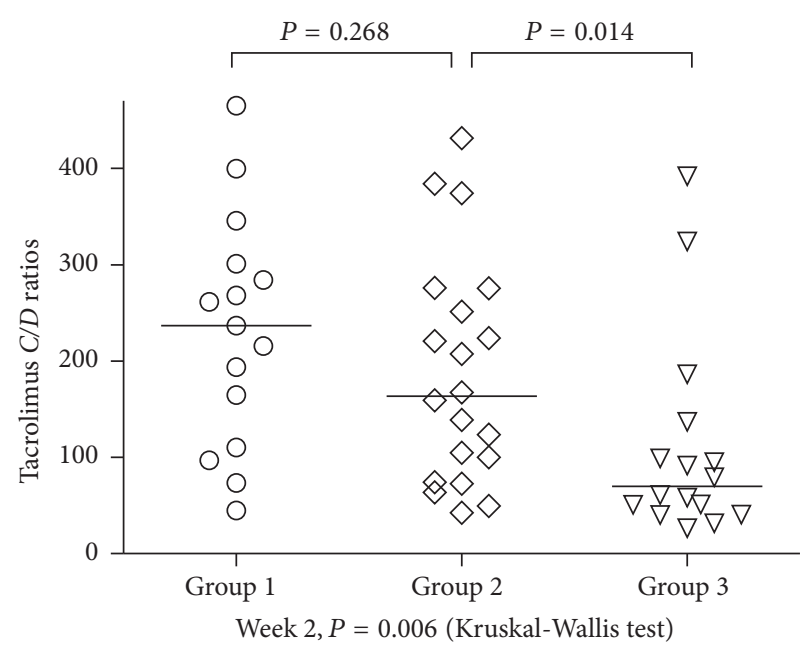

(b)

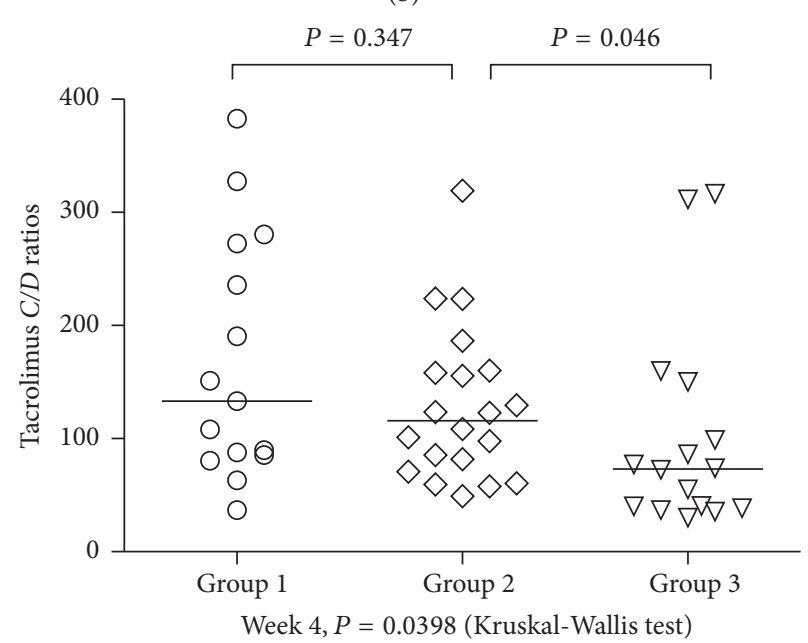

(d)

FIGURE 1: Combined effect of IL-18 rs5744247 and rs1946518 polymorphisms on tacrolimus concentration/dose (C/D) ratios in lung transplant patients at week 1 (a), week 2 (b), week 3 (c), and week 4 (d) following transplantation. IL-18 rs5744247 allele C and rs1946518 allele A were associated with fast tacrolimus metabolism. The lung transplant recipients were divided into 3 groups according to the numbers of these alleles: Group 1 (the number of alleles less than or equal to 1), Group 2 (the number of alleles equal to 2), and Group 3 (the number of alleles greater than or equal to 3). Patients who carried greater than or equal to 3 alleles associated with fast tacrolimus metabolism (Group 3 ) had lower tacrolimus $C / D$ ratios than the other groups (Kruskal-Wallis test, $P<0.05$ ).

$224.0 \pm 192.4,197.7 \pm 226.3$, and $151.0 \pm 193.5$ at week 1 , week 2 , week 3 , and week 4, respectively. For CA genotype carriers, tacrolimus $C / D$ ratios were $191.6 \pm 192.2,159.3 \pm 194.6,158.7 \pm$ 158.6, and 108.1 \pm 96.8 For AA genotype carriers, tacrolimus $C / D$ ratios were $76.6 \pm 108.8,9.2 \pm 86.3,65.9 \pm 84.4$, and $73.9 \pm 110.3$ The differences were significant at week 1 , week 2 , and week $3(P=0.007,0.018$, and 0.019 , resp.) and were nearly significant at week $4(P=0.079)$. Tacrolimus $C / D$ ratios of IL-10 rs1800896 AA genotype carriers were $193.9 \pm 224.79$, $176.8 \pm 205.14,170.8 \pm 186.69$, and $104.8 \pm 125.9$ at week 1 , week 2, week 3, and week 4, respectively. For AG genotype carriers, tacrolimus $C / D$ ratios were $77.19 \pm 66.4,74.13 \pm 32.24$, $77.84 \pm 41.57$, and $85.55 \pm 44.9$ For AA genotype carriers, tacrolimus $C / D$ ratios were $76.6 \pm 108.8,9.2 \pm 86.3,65.9 \pm 84.4$, and $73.9 \pm 110.3$ The differences were significant at week 1 and week $2(P=0.027$ and 0.034 , resp.). No significant difference of tacrolimus $C / D$ ratios of IL-10 rs1800872 and rs3021097 was found in our data.

3.2. Associations between Combined IL-18 rs5744247 and rs1946518 Polymorphisms and Tacrolimus C/D Ratios. IL18 rs5744247 and rs1946518 were shown to be associated with tacrolimus elimination. Therefore, the two genotypes were further investigated in a combination analysis. IL-18 rs5744247 allele C and rs1946518 allele A were shown to be associated with fast tacrolimus metabolism as stated above. Patients in Group 1 carried less than or equal to 1 allele; patients in Group 2 carried 2 alleles; and patients in Group 3 carried greater than or equal to 3 alleles. Group 3 had lower tacrolimus $C / D$ ratios than Group 1 and Group 2 (KruskalWallis test, $P<0.05$ ). No significant difference was found in tacrolimus $C / D$ ratios of Group 1 and Group 2 (Figure 1 ). 


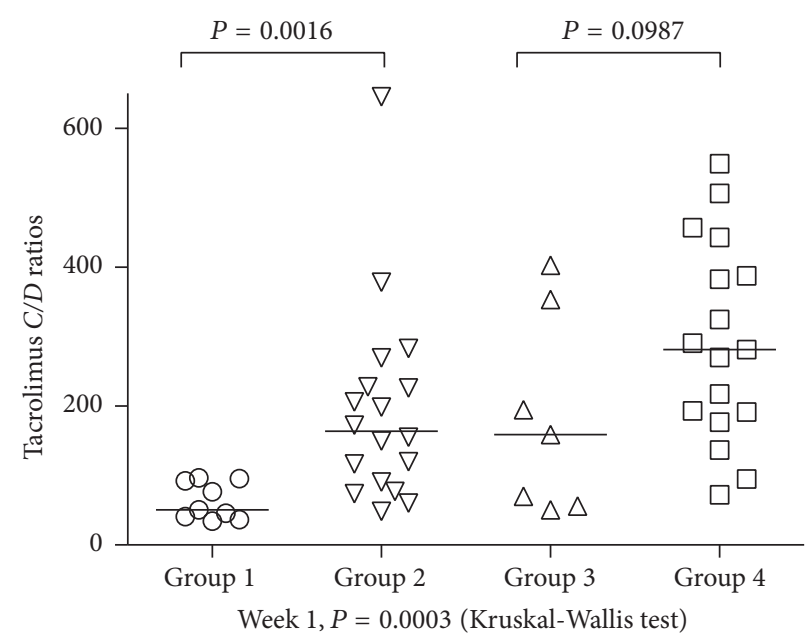

(a)

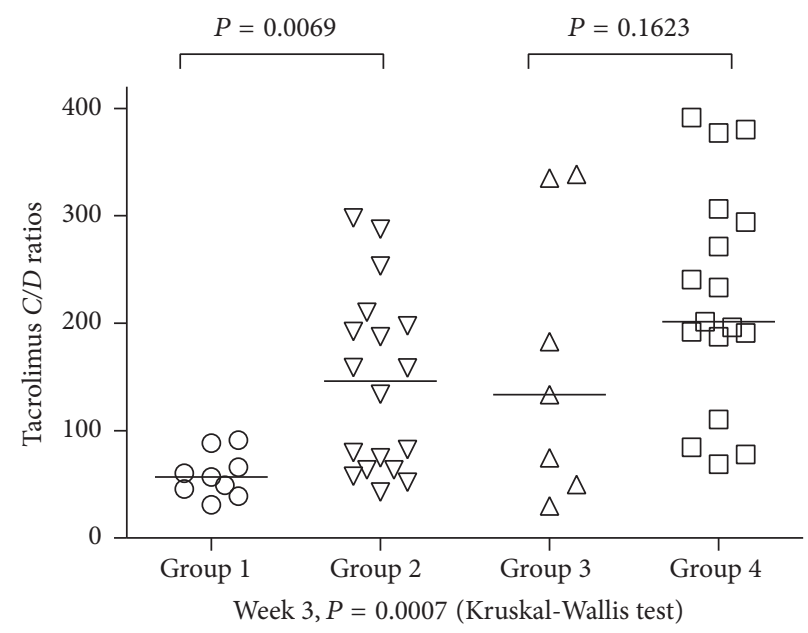

(c)

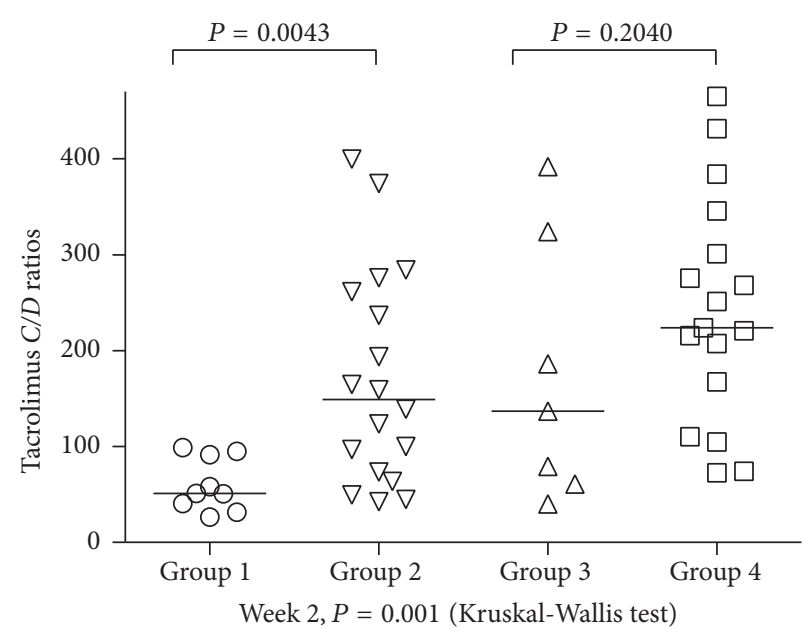

(b)

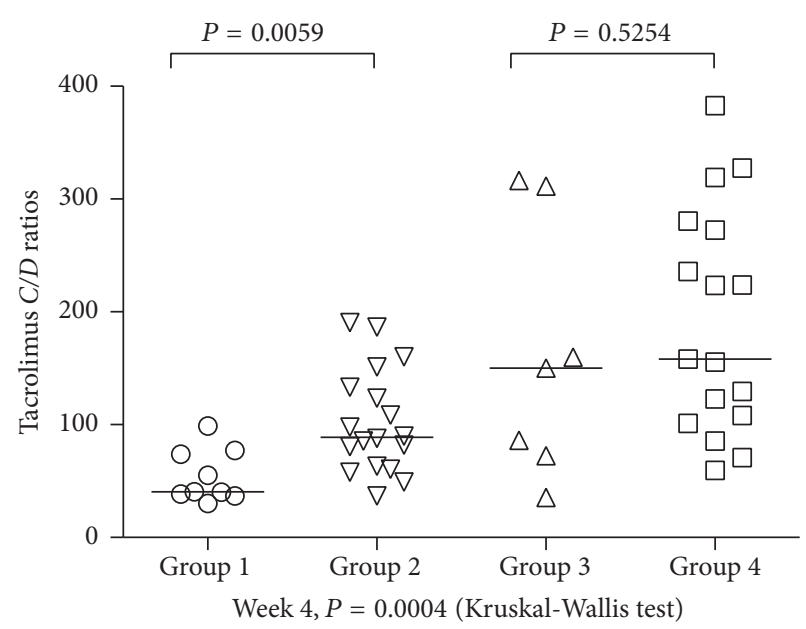

(d)

Figure 2: Combined effect of IL-18 rs5744247 and rs1946518 and CYP3A5 rs776746 polymorphisms on tacrolimus concentration/dose (C/D) ratios in lung transplant patients at week 1 (a), week 2 (b), week 3 (c), and week 4 (d) following transplantation. The lung transplant recipients were divided into 4 groups according to the number of IL-18 alleles associated with fast tacrolimus metabolism and CYP3A5 genotype: Group 1 (CYP3A5 expresser and the number of alleles greater than or equal to 3); Group 2 (CYP3A5 expressers and the number of alleles less than or equal to 2); Group 3 (CYP3A5 nonexpresser and the number of alleles greater than or equal to 3); Group 4 (CYP3A5 nonexpressers and the number of alleles less than or equal to 2). Among the patients with CYP3A5 expression genotype, the impact of IL-18 polymorphisms on tacrolimus $C / D$ ratios was significant.

3.3. Analysis of IL-18 Polymorphisms on Tacrolimus C/D Ratios in Lung Transplant Recipients Stratified by CYP3A5 Genotype. The lung transplant recipients were divided into 4 groups according to the number of IL-18 alleles associated with fast tacrolimus metabolism and CYP3A5 genotype. Patients in Group 1 were CYP3A5 expressers and carried greater than or equal to 3 alleles; patients in Group 2 were CYP3A5 expressers and carried less than or equal to 2 alleles; patients in Group 3 were CYP3A5 nonexpressers and carried greater than or equal to 3 alleles; patients in Group 4 were CYP3A5 nonexpressers and carried less than or equal to 2 alleles. Among CYP3A5 expresser recipients, the impact of IL-18 polymorphisms on tacrolimus $C / D$ ratios was significant at week 1 , week 2 , week 3 , and week $4(P=0.016,0.043$, 0.0069 , and 0.0059 , resp.). No significant influence of IL-18 polymorphisms on tacrolimus $C / D$ ratios was observed in CYP3A5 nonexpresser recipients (Figure 2).

3.4. Influence of rs5744247 and rs1946518 on IL-18 mRNA in Liver Tissues. IL-18 rs5744247 polymorphism was significantly associated with IL-18 mRNA expression (GG>CG>CC, $P=0.175)$; IL-18 rs1946518 polymorphism was significantly associated with IL-18 mRNA expression (CC $>$ CA $>$ AA, $P=$ 0.0403) (Figure 3).

\section{Discussion}

CYP3A5 rs776746 was a well-known biomarker for tacrolimus elimination [10-14]. In the present study, lung transplant recipients carrying CYP3A5 rs776746 AA/AG genotype 


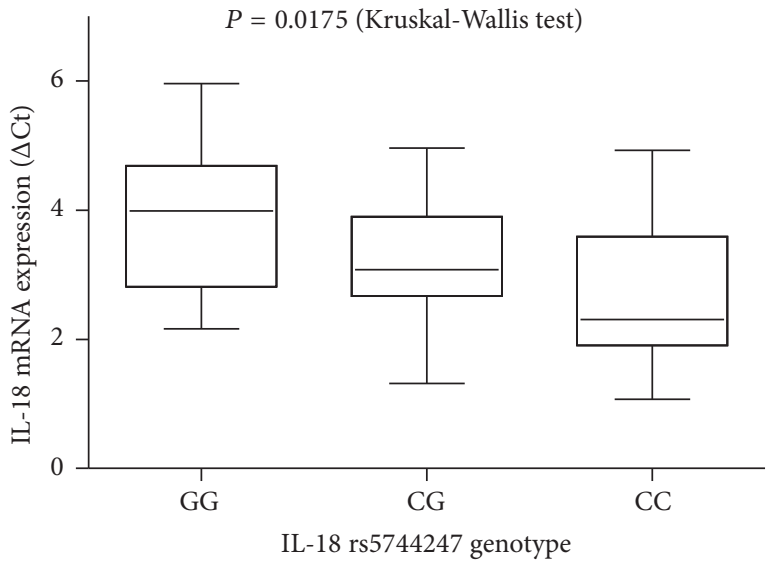

(a)

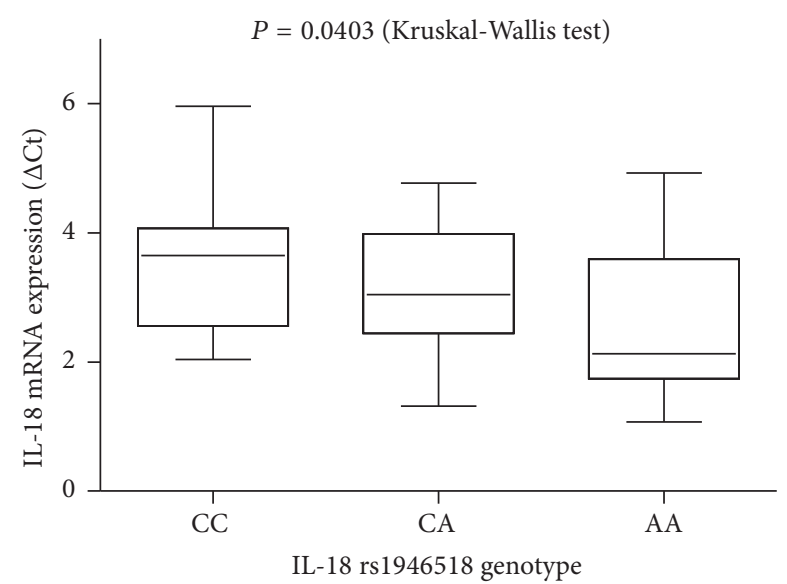

(b)

FIGURE 3: Analysis of IL-18 mRNA expressions in liver tissues with different IL-18 polymorphisms. IL-18 expression was determined by real-time PCR. IL-18 rs5744247 polymorphism was significantly associated with IL-18 mRNA expression (GG>CG>CC); IL-18 rs1946518 polymorphism was significantly associated with IL-18 mRNA expression (CC $>C A>A A)$.

had lower tacrolimus $C / D$ ratios than those carrying CYP3A5 rs776746 GG genotype. Our data confirmed that CYP3A5 rs776746 polymorphism could be used to distinguish the tacrolimus elimination phenotype in lung transplantation. However, CYP3A5 rs776746 could not be applied to guide the individual dosage regimen of tacrolimus administered in routine transplant clinical practice for two reasons. First, CYP3A5 rs776746 can only classify patients into two categories with similar proportion in the Han Chinese. Second, the $C / D$ ratios of the two categories had a large proportion of overlap [27, 28]. Further study is required to refine this process.

Rs1800896 (A-1082G), rs1800872 (A-592C), and rs3021097 (T-819C) were located in the promoter region of IL-10 and were the most IL-10 SNPs genotyped in previous studies $[29,30]$. We found that the tacrolimus C/D ratios of IL-10 rs1800896 AA genotype carriers were significantly higher than of AG carriers in the first 2 weeks after transplantation. However, the minor allele frequency (MAF) of rs1800896 was 0.068 in the present study. The SNPs with low MAF $(<5 \%)$ were usually rejected in association study [31]. As previously described, ethnicity can strongly influence the distribution of cytokine gene polymorphisms. In Caucasian patients, the IL-10 AA genotype at position -1082 occurred in $32.5 \%$ [32], while among Asian patients it occurred in $88.2 \%$ [33]. We thought the influence of rs1800896 on physiological and pathological phenomena in the Asian population had limited significance due to the low MAF of rs1800896.

In the present study, no significant difference of tacrolimus $C / D$ ratios of IL-10 rs1800872 and rs3021097 was found in our data. This was inconsistent with the previous findings that IL-10 polymorphisms influenced tacrolimus pharmacokinetics in liver and kidney transplantation [18-21]. A meta-analysis including 595 rejection patients and 1239 stable graft patients suggested that the IL-10 gene polymorphisms (including rs1800896, rs1800872, and rs3021097) were not associated with an increased graft rejection risk in kidney transplantation [34]. Another meta-analysis suggested that IL-10 rs1800896 polymorphism may not be associated with acute rejection risk in liver transplantation recipients among Caucasians [35]. Also, there is no association between IL-10 polymorphisms (rs1800872 and rs3021097) and the incidence of acute rejection after lung transplantation [36]. The change of IL-10 mRNA level during rejection episode remained controversial [34]. There was clear evidence that the IL-10 rs1800872 and rs3021097 genotypes have an association with the decreased risk of infection in lung transplant recipients [36]. Incidence rate of infection in lung transplant recipients was higher than in liver and kidney transplant recipients. Therefore, we inferred that the protective effect of IL-10 polymorphisms on infection could confuse their influence on tacrolimus pharmacokinetics.

IL-18 rs5744247 and rs1946518 were two functional polymorphisms. IL-18 rs5744247 C>G variant reflects higher transcriptional activity and higher expression of IL-18 in LPSstimulated monocytes and a higher serum IL-18 level [37]. IL-18 mRNA level significantly decreased gene expression in rs1946518 AA homozygotes (as compared with C allele carriers) in peripheral blood mononuclear cells [38]. Our data firstly showed that IL-18 rs5744247 and rs1946518 polymorphism was significantly associated with IL-18 mRNA expression in liver tissues. IL-18 rs5744247 C allele and rs1946518 A allele represented the low IL-18 mRNA expression allele. IL-18 is a member of the IL-1 family that has a homologous amino acid sequence to IL-1 $\beta$. IL- $1 \beta$ has been shown to downregulate CYP3A protein by transcriptional and posttranscriptional mechanisms [39]. It had been demonstrated that IL-18 was a negative regulator of CYP3A5 mRNA expression in hepatocytes and could influence tacrolimus metabolism in liver transplant [23]. In the present study, we firstly proposed that IL-18 rs5744247 allele C and rs1946518 allele A were associated with fast tacrolimus metabolism and 
further combined analysis showed that the numbers of low IL-18 mRNA expression alleles had positive correlation with tacrolimus $C / D$ ratios in lung transplant recipients.

In addition, our data showed that the influence of IL18 polymorphisms on tacrolimus $C / D$ ratios was observed in CYP3A5 expresser recipients, but not in CYP3A5 nonexpresser recipients.

In summary, our study suggested that $I L-18$ rs5744247 and rs1946518 genotype contribute to differences in the $C / D$ ratios of tacrolimus in lung transplantation patients. These findings may help to tailor tacrolimus administration in lung transplant recipients.

Our study has several limitations. First, the study population is confined to the Han Chinese nationality. The distribution of gene polymorphisms varies in different ethnicities. So, the conclusions of this study may not be applied to other ethnic populations. Second, this study is a single-center study and a small number of participants were involved. Further study with large sample sizes should been made to verify the association between cytokine gene polymorphisms and tacrolimus elimination.

\section{Abbreviations}

\author{
IL-18: Interleukin-18 \\ IL-10: Interleukin-10 \\ CYP: Cytochrome P450 \\ SNP: Single nucleotide polymorphism \\ $C / D$ : Serum concentration to dose ratio \\ EDTA: Ethylenediaminetetraacetic acid \\ PCR: Polymerase chain reaction.
}

\section{Competing Interests}

The authors who have taken part in this study declared that they do not have anything to disclose regarding funding or conflict of interests with respect to this manuscript.

\section{Authors' Contributions}

Gening Jiang, Junwei Fan, and Xiaoqing Zhang conceived and designed the experiments. Junwei Fan, Tao Zhang, Wei Zhang, Shengtao Lin, Ling Ye, and Yuan Liu performed the experiments. Yuping Li and Boxiong Xie analyzed the data. Xiaoqing Zhang, Junwei Fan, and Yuping Li contributed reagents, materials, and analysis tools. Junwei Fan wrote the paper. Xiaoqing Zhang and Jiandong Xu contributed equally to this work.

\section{Acknowledgments}

This work was funded by the National Natural Science Foundation of China (81202609) to Xiaoqing Zhang.

\section{References}

[1] B. A. Whitson and D. Hayes, "Indications and outcomes in adult lung transplantation," Journal of Thoracic Disease, vol. 6, no. 8, pp. 1018-1023, 2014.
[2] M. Hartert, Ö. Senbaklavaci, B. Gohrbandt, B. M. Fischer, R. Buhl, and C.-F. Vahl, "Lung transplantation: a treatment option in end-stage lung disease," Deutsches Arzteblatt International, vol. 111, no. 7, pp. 107-116, 2014.

[3] S. D. Nathan, "The future of lung transplantation," Chest, vol. 147, no. 2, pp. 309-316, 2015.

[4] C. A. Witt, V. Puri, A. E. Gelman, A. S. Krupnick, and D. Kreisel, "Lung transplant immunosuppression-time for a new approach?” Expert Review of Clinical Immunology, vol. 10, no. 11, pp. 1419-1421, 2014.

[5] J. L. Scheffert and K. Raza, "Immunosuppression in lung transplantation," Journal of Thoracic Disease, vol. 6, no. 8, pp. 1039-1053, 2014.

[6] K. Afshar, "Future direction of immunosuppression in lung transplantation," Current Opinion in Organ Transplantation, vol. 19, no. 6, pp. 583-590, 2014.

[7] L. Wang, H. L. McLeod, and R. M. Weinshilboum, "Genomics and drug response," New England Journal of Medicine, vol. 364, no. 12, pp. 1144-1153, 2011.

[8] D. A. Hesselink, R. Bouamar, L. Elens, R. H. N. Van Schaik, and T. Van Gelder, "The role of pharmacogenetics in the disposition of and response to tacrolimus in solid organ transplantation," Clinical Pharmacokinetics, vol. 53, no. 2, pp. 123-139, 2014.

[9] T. Van Gelder, R. H. Van Schaik, and D. A. Hesselink, "Practicability of pharmacogenetics in transplantation medicine," Clinical Pharmacology and Therapeutics, vol. 95, no. 3, pp. 262264, 2014.

[10] S. S. Nair, S. Sarasamma, N. Gracious, J. George, T. S. N. Anish, and R. Radhakrishnan, "Polymorphism of the CYP3A5 gene and its effect on tacrolimus blood level," Experimental and Clinical Transplantation, vol. 13, pp. 197-200, 2015.

[11] L. E. Rojas, M. J. Herrero, V. Bosó et al., "Meta-analysis and systematic review of the effect of the donor and recipient CYP3A5 6986A $>$ G genotype on tacrolimus dose requirements in liver transplantation," Pharmacogenetics and Genomics, vol. 23, no. 10, pp. 509-517, 2013.

[12] S. Z. Vahed, M. Ardalan, N. Samadi, and Y. Omidi, "Pharmacogenetics and drug-induced nephrotoxicity in renal transplant recipients," BioImpacts, vol. 5, no. 1, pp. 45-54, 2015.

[13] C. Monchaud, B. C. De Winter, C. Knoop et al., "Population pharmacokinetic modelling and design of a bayesian estimator for therapeutic drug monitoring of tacrolimus in lung transplantation," Clinical Pharmacokinetics, vol. 51, no. 3, pp. 175-186, 2012.

[14] H. Zheng, A. Zeevi, E. Schuetz et al., "Tacrolimus dosing in adult lung transplant patients is related to cytochrome P4503A5 gene polymorphism," Journal of Clinical Pharmacology, vol. 44, no. 2, pp. 135-140, 2004.

[15] A. Provenzani, A. Santeusanio, E. Mathis et al., "Pharmacogenetic considerations for optimizing tacrolimus dosing in liver and kidney transplant patients," World Journal of Gastroenterology, vol. 19, no. 48, pp. 9156-9173, 2013.

[16] C.-M. Lee, J. Pohl, and E. T. Morgan, "Dual mechanisms of CYP3A protein regulation by proinflammatory cytokine stimulation in primary hepatocyte cultures S," Drug Metabolism and Disposition, vol. 37, no. 4, pp. 865-872, 2009.

[17] A. Westerholt, S. Himpel, B. Hager-Gensch et al., "Intragraft iNOS induction during human liver allograft rejection depresses cytochrome p450 activity," Transplant International, vol. 17, no. 7, pp. 370-378, 2004. 
[18] D. Li, J.-Y. Zhu, J. Gao, X. Wang, Y.-Q. Lou, and G.-L. Zhang, "Polymorphisms of tumor necrosis factor- $\alpha$, interleukin-10, cytochrome P450 3A5 and ABCB1 in Chinese liver transplant patients treated with immunosuppressant tacrolimus," Clinica Chimica Acta, vol. 383, no. 1-2, pp. 133-139, 2007.

[19] D. Li, W. Lu, J.-Y. Zhu, J. Gao, Y.-Q. Lou, and G.-L. Zhang, "Population pharmacokinetics of tacrolimus and CYP3A5, MDR1 and IL-10 polymorphisms in adult liver transplant patients," Journal of Clinical Pharmacy and Therapeutics, vol. 32, no. 5, pp. 505-515, 2007.

[20] X. Zhang, Z. Wang, J. Fan, G. Liu, and Z. Peng, "Impact of interleukin-10 gene polymorphisms on tacrolimus dosing requirements in Chinese liver transplant patients during the early posttransplantation period," European Journal of Clinical Pharmacology, vol. 67, no. 8, pp. 803-813, 2011.

[21] C.-J. Li, L. Li, L. Lin et al., "Impact of the CYP3A5, CYP3A4, COMT, IL-10 and POR genetic polymorphisms on tacrolimus metabolism in Chinese renal transplant recipients," PLoS ONE, vol. 9, no. 1, Article ID e86206, 2014.

[22] Y. Li, Y. Zou, B. Cai et al., "The associations of IL-18 serum levels and promoter polymorphism with tacrolimus pharmacokinetics and hepatic allograft dysfunction in Chinese liver transplantation recipients," Gene, vol. 491, no. 2, pp. 251-255, 2012.

[23] J. Fan, X. Zhang, L. Ren et al., "Donor IL-18 rs5744247 polymorphism as a new biomarker of tacrolimus elimination in Chinese liver transplant patients during the early post-transplantation period: results from two cohort studies," Pharmacogenomics, vol. 16, no. 3, pp. 239-250, 2016.

[24] J. Xing, X. Zhang, J. Fan, B. Shen, T. Men, and J. Wang, "Association between interleukin-18 promoter variants and tacrolimus pharmacokinetics in Chinese renal transplant patients," European Journal of Clinical Pharmacology, vol. 71, no. 2, pp. 191-198, 2015.

[25] Z. Li, Z. Zhang, Z. He et al., "A partition-ligation-combinationsubdivision em algorithm for haplotype inference with multiallelic markers: Update of the SHEsis (http://analysis.bio- x.cn)," Cell Research, vol. 19, no. 4, pp. 519-523, 2009.

[26] S. Gatault, M. Delbeke, V. Driss et al., "IL-18 is involved in eosinophil-mediated tumoricidal activity against a colon carcinoma cell line by upregulating LFA-1 and ICAM-1," The Journal of Immunology, vol. 195, no. 5, pp. 2483-2492, 2015.

[27] L. E. Rojas, M. J. Herrero, V. Bosó et al., "Meta-analysis and systematic review of the effect of the donor and recipient CYP3A5 6986A $>\mathrm{G}$ genotype on tacrolimus dose requirements in liver transplantation," Pharmacogenetics and Genomics, vol. 23, no. 10, pp. 509-517, 2013.

[28] H. de Jonge and D. R. J. Kuypers, "Pharmacogenetics in solid organ transplantation: current status and future directions," Transplantation Reviews, vol. 22, no. 1, pp. 6-20, 2008.

[29] T.-C. Hsia, W.-S. Chang, S.-J. Liang et al., "Interleukin-10 (IL10) promoter genotypes are associated with lung cancer risk in Taiwan males and smokers," Anticancer Research, vol. 34, no. 12, pp. 7039-7044, 2014.

[30] W.-H. Kuo, C.-Y. Huang, C.-K. Fu et al., "Effects of interleukin10 polymorphisms and smoking on the risk of gastric cancer in Taiwan," In Vivo, vol. 28, no. 5, pp. 967-972, 2014.

[31] S. Lee, G. R. Abecasis, M. Boehnke, and X. Lin, "Rarevariant association analysis: study designs and statistical tests," American Journal of Human Genetics, vol. 95, no. 1, pp. 5-23, 2014.
[32] V. R. Mas, R. A. Fisher, D. G. Maluf et al., "Polymorphisms in cytokines and growth factor genes and their association with acute rejection and recurrence of hepatitis $C$ virus disease in liver transplantation," Clinical Genetics, vol. 65, no. 3, pp. 191201, 2004.

[33] H. Y. Xie, W. L. Wang, M. Y. Yao et al., "Polymorphisms in cytokine genes and their association with acute rejection and recurrence of hepatitis B in Chinese liver transplant recipients," Archives of Medical Research, vol. 39, no. 4, pp. 420-428, 2008.

[34] J. Xiong, Y. Wang, Y. Zhang et al., "Lack of association between interleukin-10 gene polymorphisms and graft rejection risk in kidney transplantation recipients: a meta-Analysis," PLoS ONE, vol. 10, no. 6, Article ID e0127540, 2015.

[35] F. Liu, B. Li, W.-T. Wang et al., "Interleukin-10-1082G/A polymorphism and acute liver graft rejection: a meta-analysis," World Journal of Gastroenterology, vol. 18, no. 8, pp. 847-854, 2012.

[36] H.-J. Mu, P. Xie, J.-Y. Chen et al., "Association of TNF- $\alpha$, TGF- $\beta 1$, IL-10, IL- 6 , and IFN- $\gamma$ gene polymorphism with acute rejection and infection in lung transplant recipients," Clinical Transplantation, vol. 28, no. 9, pp. 1016-1024, 2014.

[37] M. Harada, K. Obara, T. Hirota et al., "A functional polymorphism in IL-18 is associated with severity of bronchial asthma," American Journal of Respiratory and Critical Care Medicine, vol. 180, no. 11, pp. 1048-1055, 2009.

[38] V. Dziedziejko, M. Kurzawski, E. Paczkowska, B. Machalinski, and A. Pawlik, "The impact of IL18 gene polymorphisms on mRNA levels and interleukin-18 release by peripheral blood mononuclear cells," Postepy Higieny i Medycyny Doswiadczalnej, vol. 66, pp. 409-414, 2012.

[39] H. Tsutsui and K. Nakanishi, "Immunotherapeutic applications of IL-18," Immunotherapy, vol. 4, no. 12, pp. 1883-1894, 2012. 


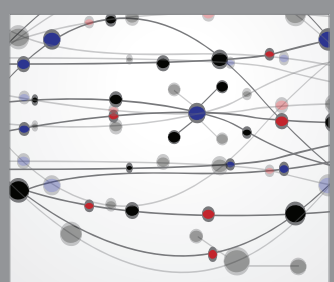

The Scientific World Journal
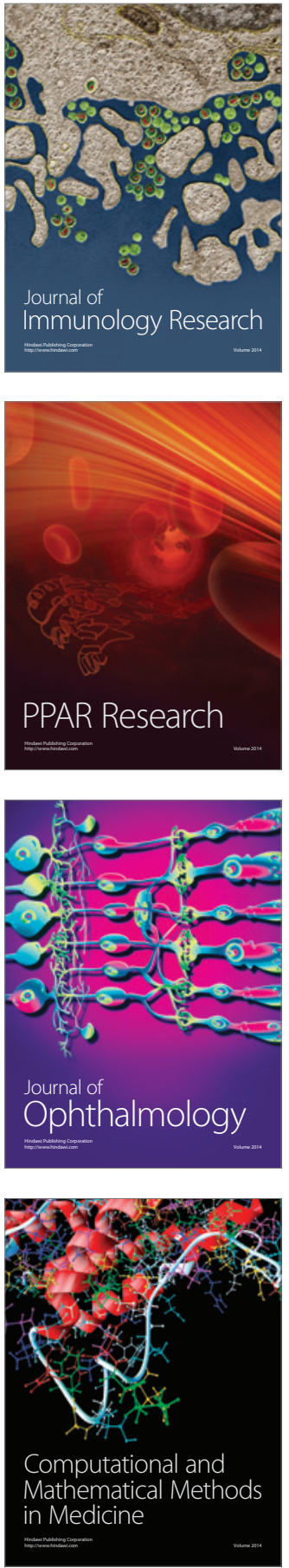

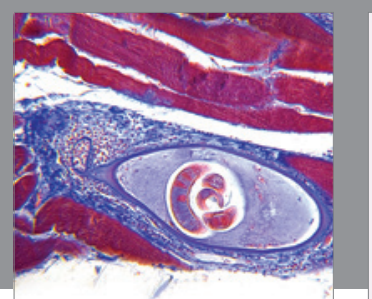

Gastroenterology Research and Practice
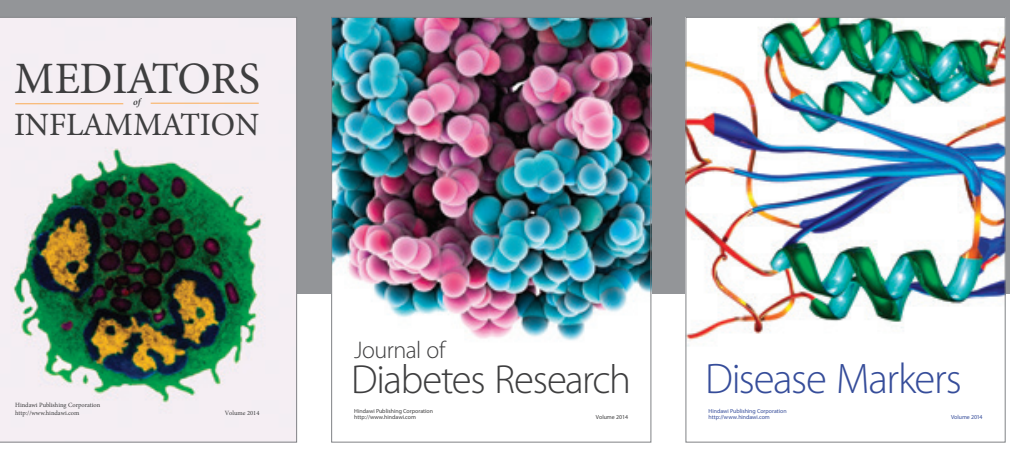

Disease Markers

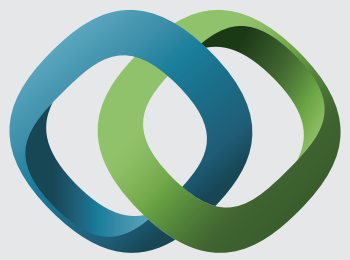

\section{Hindawi}

Submit your manuscripts at

https://www.hindawi.com
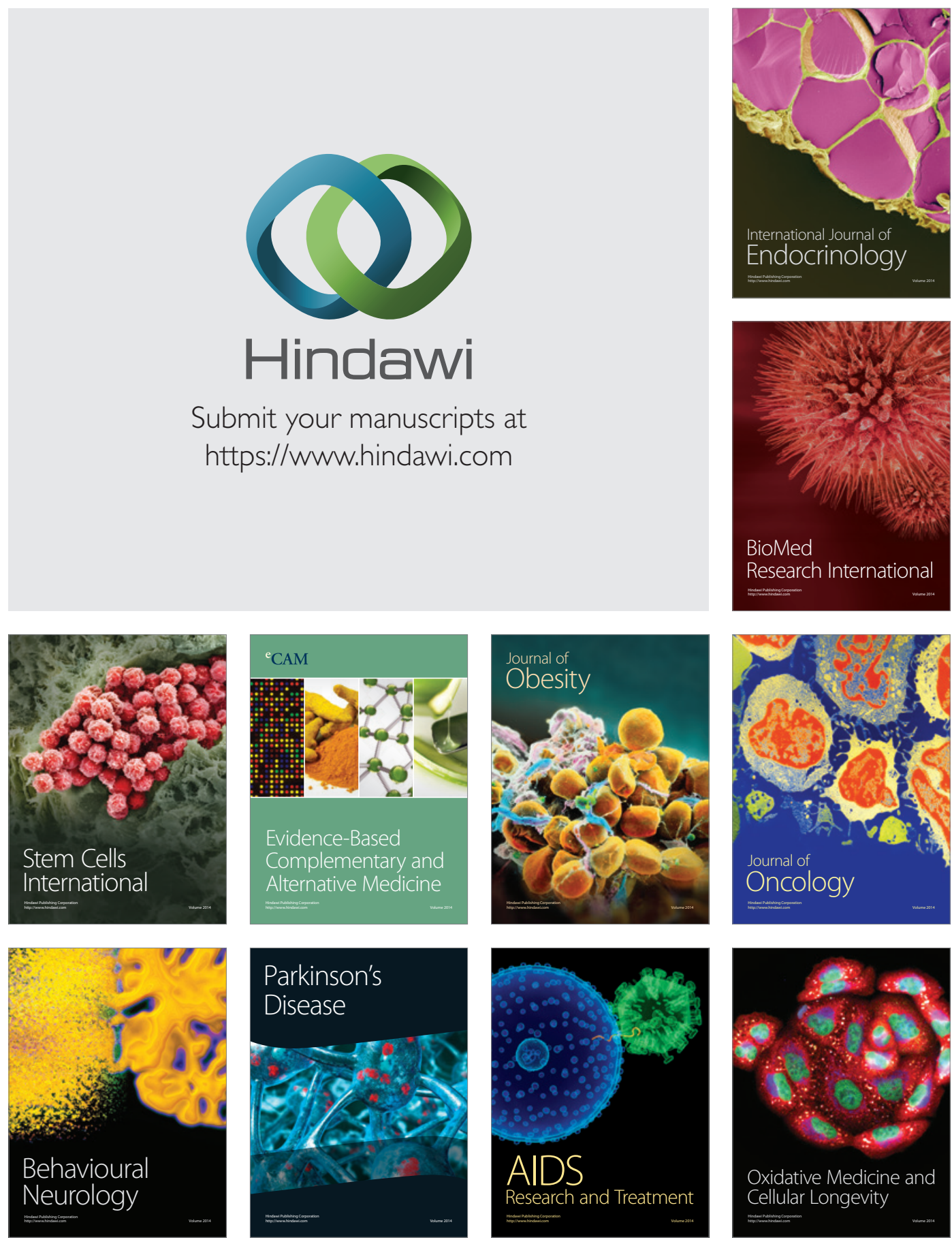